\title{
Editorial: change of Editor
}

This is the first number of the Annals in 11 years which has gone to press not under the editorship of Dr Tom Scott. It brings to an end a stewardship marked by sound and wise editorial guidance. All who read the journal, write for it, or produce it owe Dr Scott a deep debt of gratitude.

There have been few changes in editorship since the Annals first appeared in 1939. The late Dr Will Copeman, effectively editor from the beginning, held this office until his death in 1971. He was helped increasingly by $\mathrm{Dr}$ Oswald Savage, upon whose retirement in $1968 \mathrm{Dr}$ Scott became assistant editor, then editor in 1971 . Throughout the life of the journal editorial policies have remained broadly consistent, attempting to serve the interests of both clinical rheumatology and related laboratory disciplines. With a new hand at the helm there will be no dramatic change of course. This is, however, a time to review editorial strategy, and there is perhaps one matter which calls for particular consideration at present, namely the delay in publication of articles.
In recent years increasing numbers of first-rate articles have been submitted for publication. To try to choose the best of these and at the same time to provide a variety that will appeal to as many readers as possible has presented the Editor with a task of some difficulty. Increases from time to time in the numbers of pages in the Annals have helped, but there is an economic limit to that solution. The result has been a gradual increase in the numbers of accepted papers awaiting publication and hence a lengthening of the period before they appear. On the whole earlier publication is desirable for many of the papers, and recently a further increase in the number of pages was arranged to help reduce the delay. The aim will be to attain prompter publication in the near future, but not at the expense of turning down good papers. Against this background succinctness of expression will more than ever count favourably in their evaluation. 